\title{
Self-assembled interpenetrating networks by orthogonal self assembly of surfactants and hydrogelators
}

\author{
Aurelie M. Brizard, ${ }^{a}$ Marc C. A. Stuart ${ }^{b}$ and Jan H. van Esch ${ }^{* a}$
}

Received 24th February 2009, Accepted 1st April 2009

First published as an Advance Article on the web 27th July 2009

DOI: 10.1039/b903806j

Interpenetrating networks (IPN) consist of two or more networks of different components which are entangled on a molecular scale and cannot be separated without breaking at least one of the networks. They are of great technological interest because they allow the blending of two or more otherwise incompatible properties or functions, and furthermore synergistic effects might arise from the simultaneous operation of the two networks. So far, the preparation of interpenetrating network gels by self-assembly approaches was doomed to fail because the conventional polymers and surfactant building blocks either phase separate or form mixed assemblies, respectively. Here we report on self-assembled interpenetrating networks obtained by the orthogonal self-assembly of small molecular hydrogelators and surfactants. Preliminary studies on the self-assembly behaviour and viscoelastic properties of these systems revealed that these self-assembled IPN have a number of intriguing properties. For instance, the presence of two coexisting networks offers new possibilities for compartmentalization, and will allow one to adjust the viscoelastic properties between 'soft' and 'hard' gels. The non-covalent character of such IPN makes their formation fully reversible, which can be exploited for dual responsive systems. Most interestingly, self-assembled IPN can also act as a very primitive, yet unique, model for biological interpenetrating networks like the extracellular matrix and the cytoskeleton, and thereby contribute to our understanding of these very complex systems.

\section{Introduction}

The self-assembly of small molecular building blocks is an attractive approach for the construction of nano-objects and nano-structured materials because their spontaneous and reversible formation under thermodynamic equilibrium conditions allows simple and large scale manufacturing methods compared to common topdown techniques. ${ }^{1}$ Over the past decade, the self-assembly approach has been applied to many different building blocks, ranging from small molecules, proteins, nano-particles and colloids, up to even mesoscale building blocks, thereby enabling the fabrication of objects and materials with regular features from nano- to micrometre dimensions. ${ }^{2}$ Among the building blocks available, surfactants which share a common structure consisting of a hydrophilic segment and a hydrophobic segment, have been extensively used because they lead to well defined basic

${ }^{a}$ Self-Assembling Systems, Department of Chemical Engineering, Delft University of Technology, Julianalaan 136, 2628 BL, Delft, The Netherlands.E-mail: j.h.vanesch@tudelft.nl ${ }^{b}$ Groningen Biomolecular Sciences and Biotechnology Institute, University of Groningen, Nijenborg 4, 9747 AG, Groningen, The Netherlands

This journal is @ The Royal Society of Chemistry 2009 Faraday Discuss., 2009, 143, 345-357 | 345 
supramolecular architectures, like spherical or rod-like micelles, bilayers, vesicles or inverted micelles. ${ }^{3}$ These aggregates encompass a large range of length scales $(2 \mathrm{~nm}$ up to several $\mu \mathrm{m}$ ), can easy integrate functional molecules due to the relatively low specificity of the hydrophobic interactions, and may encapsulate an aqueous compartment, which has led to widespread applications as detergents, catalysis, structuring agents, templates, and drug delivery. ${ }^{4}$ Despite such diverse applications, surfactants however suffer from the limited scope of supramolecular architectures they can generate, justifying the need for the development of other building blocks. Small molecule gelators, peptides, block copolymers, and polymer-protein conjugates, have thus been used to construct nano-scale assemblies in aqueous environments, leading to novel architectures like ribbons, helices and tubes. ${ }^{5,6}$ Considerable progress has even been made in controlling the diameter of 1-D aggregates, e.g. via the tuning of the chiral twist, ${ }^{7}$ or adding functionality by incorporating biological entities or sensors for instance. ${ }^{8}$

This tremendous increase in the diversity of available molecular building blocks, however, does not allow the level of complexity or functionality found in nature to be met or the needs of future nanotechnology to be complied with. Interestingly, in nature, complex processes like replication, transcription, energy storage and several metabolic pathways rely more on the separation of incompatible structures in space and time, rather than on the diversity of the buildings blocks, which remain relatively limited. In cells for instance, these processes are achieved through compartmentalization by use of bilayer membranes, however always in conjunction with an internal cytoskeleton or an external cell wall. Remarkably, all these objects are self-assembled structures that can coexist because self-assembly of each individual constituent does not interfere with the others. It is this ability to self-sort and the tight integration of these self-assembled structures that gives cells their stability, shape and function.

The ability to integrate different structural elements is also essential for the fabrication of nano-structures with regular features in the $10-1000 \mathrm{~nm}$ regime. ${ }^{2}$ These materials are expected to have superior mechanical properties like bone and wood, and are indispensable for photonic applications. The step-wise self-assembly of small components to such hierarchically structured objects and materials is also common in nature, like with viruses and ribosomes but also tissues such as bone and wood, and is receiving increasing attention as a valid approach to the fabrication of more complex nanostructures and materials. ${ }^{9}$ Its successful application to generate well-defined objects or regular materials, however, requires tight control over the self-assembly process with regard to shape and size but also the strength and nature of the interactions between the different entities at each hierarchical level.

There is thus an urgent need to extend the current toolbox of available nanoarchitectures that can be obtained by self-assembly of structurally simple compounds. Just like in nature, a straightforward approach to increase the level of complexity in self-assembling systems consists of making use of multiple components that can display orthogonal self assembly, i.e. the independent formation of two supramolecular structures each with their own characteristic within a single system. Remarkably, the controlled phase separation between multiple different components has only recently been exploited to fabricate assemblies and materials with regular features at nanolength scale, i.e. nanostructure formation and interfacial patterning in phase separated polymer systems, ${ }^{9,10}$ and low molecular weight organogels in liquid crystalline phases. ${ }^{11}$

Recently, we showed that the orthogonal self-assembly of surfactant molecules and small molecular hydrogelators is a versatile and powerful approach towards the formation of novel and more complex architectures. ${ }^{12}$ For example, the orthogonal self-assembly of hydrogelators with micelle forming surfactants or phospholipids leads to the formation of micelles embedded in gel matrixes or vesicles encapsulated in a gel, respectively (Fig. 1). This last example has been exploited to successfully develop liposomes with an encapsulated self-assembling hydrogel

346 | Faraday Discuss., 2009, 143, 345-357 This journal is @ The Royal Society of Chemistry 2009 


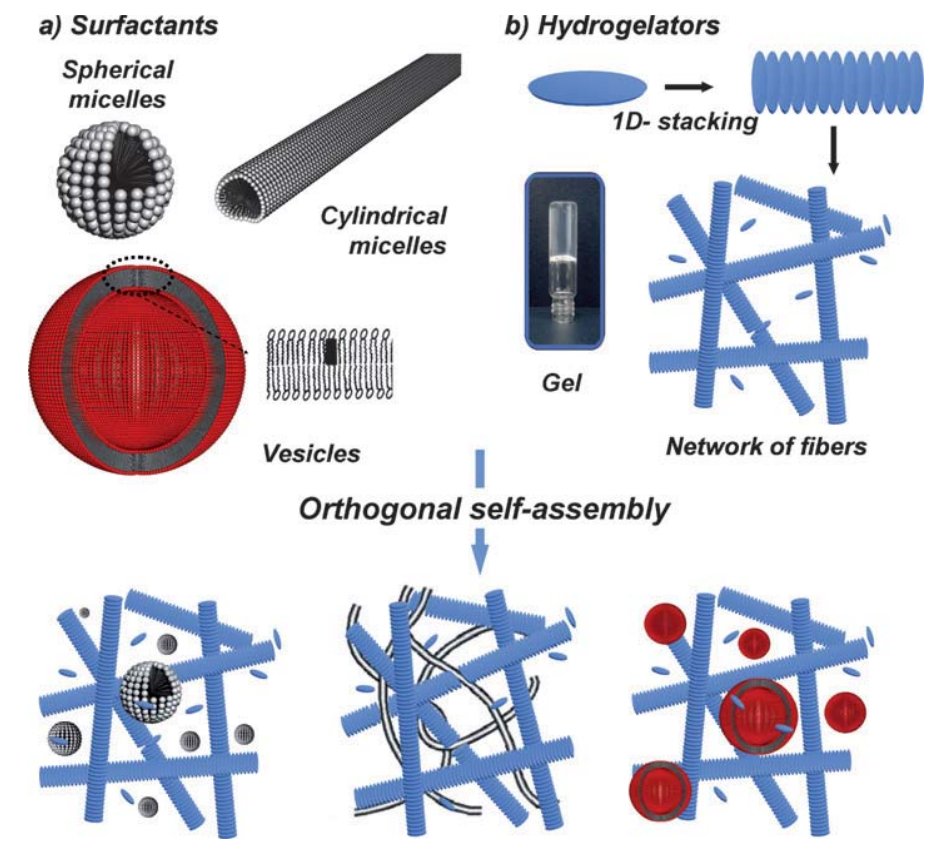

Fig. 1 Schematic illustration of orthogonal self-assembly between surfactants-which can form a variety of structures such as spherical micelles, wormlike micelles or vesicles - and hydrogelators, known to self-assemble in one dimension to give a network of fibers. Orthogonal self-assemblies between these two types of entities allows the independent formation of gel fibers in conjunction with micelles or vesicles.

("gellosomes"). The highly responsive character of the gelator makes it very attractive not only as a mimic of cytoskeleton ${ }^{13}$ but also for drug delivery purposes.

Another system that is of great potential interest consists of self-assembled interpenetrating networks (IPN). Interpenetrating networks ${ }^{14}$ consist of two or more networks of different components which are entangled on a molecular scale and cannot be separated without breaking at least one of the networks. They have been known for decades and are of great technological interest because they allow the blending of two or more otherwise incompatible properties or functions, and furthermore synergistic effects might arise from the simultaneous operation of the two networks. ${ }^{15}$ So far, the preparation of interpenetrating network gels by selfassembly approaches was doomed to fail because the conventional polymers and surfactant building blocks either phase separate or form mixed assemblies, respectively. Recently, we found that the orthogonal self-assembly of low molecular weight (LMW) gelators together with surfactants allows the preparation of interpenetrating networks exclusively by self-assembly. ${ }^{12}$ Here we report on our ongoing work on the self-assembly properties together with preliminary results on the viscoelastic properties of a prototype self-assembled IPN formed from a low molecular weight hydrogelator and a cylindrical micelle forming surfactant.

\section{Experimental}

\section{Materials}

Cetyltrimethylammonium tosylate (CTAT) was purchased from Sigma Aldrich and used without any further purification. The synthesis and full characterization of compound HG1 has been reported before. ${ }^{16}$

This journal is ( ) The Royal Society of Chemistry 2009 Faraday Discuss., 2009, 143, 345-357 | 347 


\section{Gel preparation}

CTAT is soluble in water above $23{ }^{\circ} \mathrm{C} \pm 1{ }^{\circ} \mathrm{C}$. At lower temperatures, CTAT exhibits a white appearance due to the presence of CTAT crystals dispersed in a viscous surfactant solution.

The gel samples were prepared by heating the gelator (typically 1 to $10 \mathrm{mM}$ ) in $1 \mathrm{~mL}$ of water or an aqueous solution containing the surfactants (binary systems) in a closed vial until a clear solution was obtained. Cooling to room temperature caused the formation of gels

\section{Gel-to-sol transition temperature determination}

All gel-to-sol transition temperatures $\left(T_{\mathrm{GS}}\right)$ were determined using the dropping ball method, which consists in carefully placing a stainless steel ball $(65 \mathrm{mg}, 2.5 \mathrm{~mm}$ in diameter) on top of a gel that had been prepared $8 \mathrm{~h}$ earlier in a $2 \mathrm{~mL}$ glass vial. These vials are subsequently placed in a heating block where the gel melting can be monitored by means of a CCD camera. The temperature of the heating block is increased by $5^{\circ} \mathrm{C} \mathrm{h}^{-1}$ and the $T_{\mathrm{GS}}$ is defined as the temperature at which the steel ball reaches the bottom of the vial.

\section{Conductivity experiments}

The micellization of CTAT in pure water or in a gel network of HG1 was studied by determining the concentration dependence of the specific conductivity at $25{ }^{\circ} \mathrm{C}$. The conductivity measurements were carried out with a CONSORT C830 conductimeter, which was first calibrated with a $0.01 \mathrm{M} \mathrm{KCl}$ standard. For the conductivity experiments in an HG1 network $(2 \mathrm{mM})$, each data point was obtained from a separate gel-CTAT mixture prepared by heating above the gel-sol transition of HG1 and subsequent cooling. To prevent any counter-ion effects, the HG1 powder was washed several times with double distilled water and freeze dried, prior to gel preparation. After this treatment, a value of $7.8 \mu \mathrm{S} \mathrm{cm}^{-1}$ was found in a $2 \mathrm{mM}$ HG1 gel in pure water, instead of $1.4 \mu \mathrm{S} \mathrm{cm}-1$ for water only. The difference $\left(6.4 \mu \mathrm{S} \mathrm{cm}^{-1}\right)$ was therefore subtracted from each measurement for the binary systems.

\section{CryoTEM}

A few microlitres of suspension were deposited on a Quantifoil 3.5/1 holey carbon coated grid. After blotting away the excess of liquid the grids were plunged quickly in liquid ethane. Frozen-hydrated specimens were mounted in a cryo-holder (Gatan, model 626) and observed in a Philips CM 120 electron microscope, operating at $120 \mathrm{kV}$. Micrographs were recorded under low-dose conditions on a slow-scan CCD camera (Gatan, model 794).

\section{Rheology}

Oscillatory experiments were performed using a rheometer (AR G2, TA instruments) in a strain controlled mode, equipped with a steel cone-and-plate geometry of $2.0^{\circ}$ and $40 \mathrm{~mm}$ in diameter. The temperature of the plates was controlled at $25^{\circ} \mathrm{C}$ with an error of $\pm 0.2^{\circ} \mathrm{C}$. In case of the pure CTAT samples, the linear regime existed up to $10 \%$ strain (strain sweep at $10 \mathrm{rad} \mathrm{s}^{-1}$ ). For HG1 gels, the viscoelastic response was linear until $1 \%$ strain only. As a consequence, all the experiments were performed with a $0.5 \%$ strain, to ensure linearity of the viscoelastic response for the binary mixtures. The rheograms of CTAT solutions were highly reproducible, while the injection method influenced the results more with the HG1 gels. The best results were obtained by first ageing the gel for at least $24 \mathrm{~h}$, pipette it slowly and inject it as such on the plate. All the experiments were performed between $0.005 \mathrm{~Hz}$ and $10 \mathrm{~Hz}$, in a stiff bearing mode.

348 | Faraday Discuss., 2009, 143, 345-357 This journal is @) The Royal Society of Chemistry 2009 


\section{Results and discussion}

In the present study, a low molecular weight gelator molecule HG1 based on 1,3,5cyclohexyltricarboxamide was used because these molecules self-assemble into 1D arrays stabilized by both hydrogen bonding and hydrophobic interactions (Fig. 2). ${ }^{16}$ A similar set of interactions serves to stabilize protein secondary structures, structures which are stable in the presence of weakly interacting surfactant. Previously it was shown that these compounds can be functionalized at the periphery with different solvophilic or $\mathrm{pH}$ sensitive groups, giving access to modular architectures and properties. In the case of HG1, hydrophilic oligoethylene oxide groups are attached leading to a nonionic hydrogelator.

Hydrogelator HG1 is insoluble in water at room temperature but gradually dissolves upon heating. Cooling of the hot solutions containing typically $0.1-1 \%$ $\mathrm{w} / \mathrm{v}$ of gelator to room temperature leads to the formation of hydrogels, with a transparent to opalescent appearance depending on their concentration. This process can be repeated many times indicating that hydrogel formation by HG1 is thermoreversible. Cryo transmission electron microscopy (TEM) showed that HG1 self-assembles into thin fibers from about $500 \mathrm{~nm}$ up to several micrometres long, and with diameters between $5 \mathrm{~nm}$ to $25 \mathrm{~nm}$ (Fig. 3a). The long aspect ratio and polydispersity with regard to width and length indicate that fiber formation is the result of a kinetically controlled, highly anisotropic growth process. ${ }^{5}$

Remarkably, the fibers have different appearances depending on the processing conditions. Samples that are prepared simply by cooling from hot isotropic solutions consist of fibrils without fine structure (Fig. 3b,c). However, after mechanical agitation (vortexing) small bands of equally spaced stripes parallel to the fiber axis are clearly visible. Macroscopically, this mechanical agitation also leads to more fragile gels, which reform very slowly at high concentration (10 mM for instance) but are irreversibly disrupted close to the critical gelation concentration $(0.4 \mathrm{mM})$. The width of a single stripe amounts to $4-5 \mathrm{~nm}$ which is in good agreement with the diameter of a single molecule of HG1. The striped bands appear at

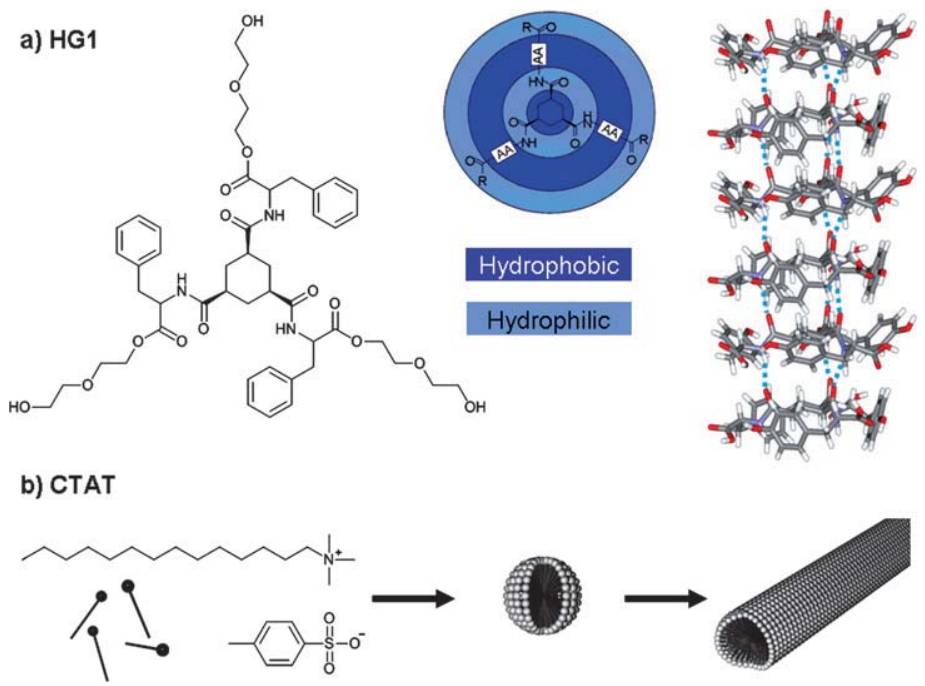

Fig. 2 Chemical structures of the molecules under study: (a) 1,3,5-triamide cis,cis-cyclohexane-based hydrogelator HG1 which self-assembles preferentially in one dimension. In the schematic representation, light blue regions correspond to hydrophilic groups and dark blue areas to hydrophobic entities ( $\mathrm{AA}=$ amino acids). (b) Cetyltrimethylammonium tosylate surfactant (CTAT). This surfactant aggregates first in spherical micelles which transform into cylindrical micelles upon increasing concentration.

This journal is @ The Royal Society of Chemistry 2009 Faraday Discuss., 2009, 143, 345-357 | 349 

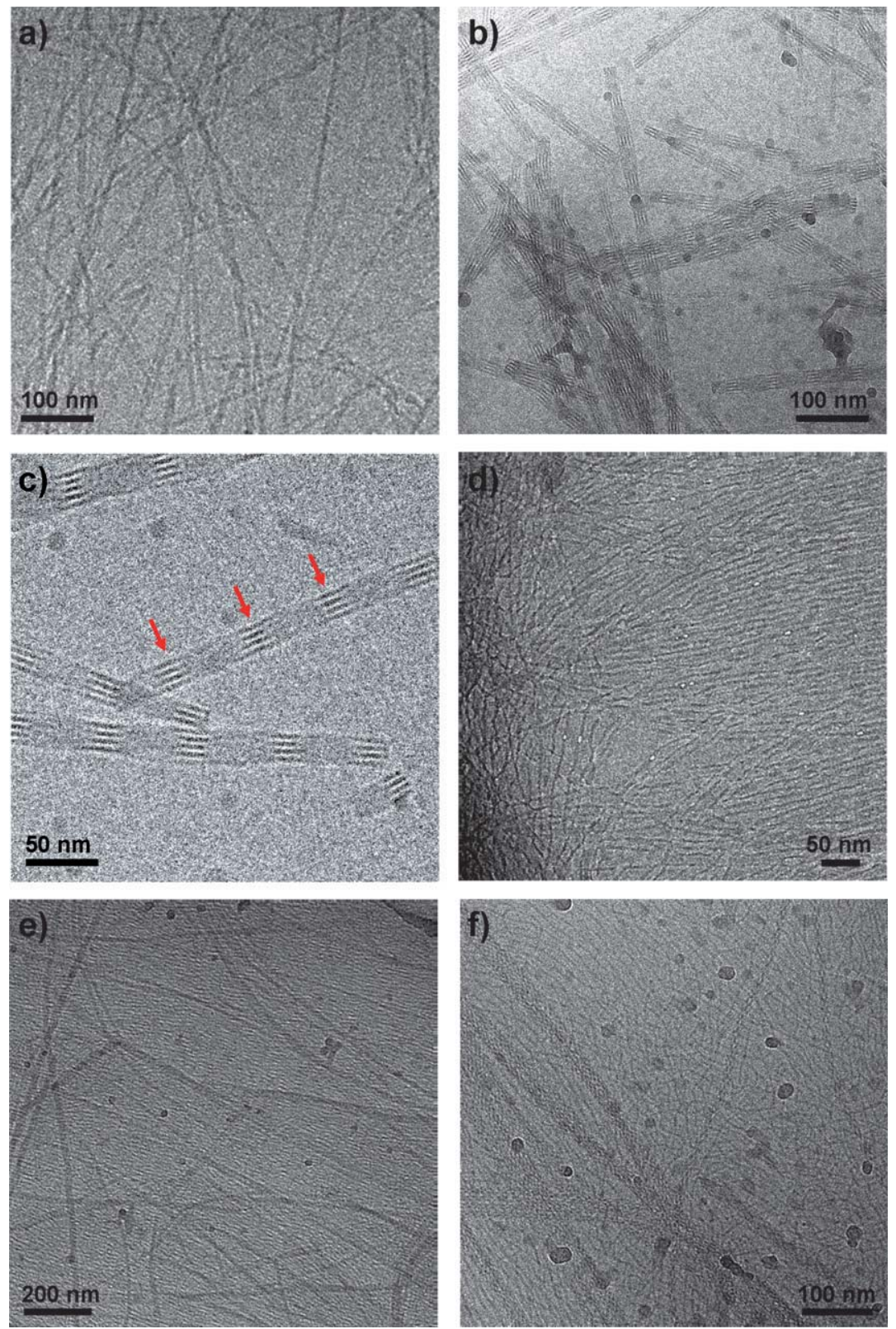

Fig. 3 CryoTEM pictures of fibrous networks of gelator HG1 (2mM) in water, (a) without vortexing or (b) and (c) after vortexing, which leads to the appearance of shorter fibers with striped bands. (d) Wormlike micelles of CTAT at $100 \mathrm{mM}$ in water. (e) and (f) Binary system of CTAT at $100 \mathrm{mM}$ and $\mathrm{HG} 1$ at $2 \mathrm{mM}$ in water.

intervals of $60 \mathrm{~nm}$ which is about 300 times the hydrogen bonded stacking distance of 1,3,5-cyclohexyltricarboxamides. ${ }^{16}$ Presumably, the fibers consist of twisted bundles of stacked molecules of HG1.

The persistence length of the fibers is much larger than their diameter and up to several hundreds of nanometres, and mechanical agitation of the samples results in the appearance of many broken fibers rather then bent ones, suggesting that

350 | Faraday Discuss., 2009, 143, 345-357 This journal is @ The Royal Society of Chemistry 2009 
they are rather stiff and brittle (Fig. 3b). Another striking observation is that these stripped bundles of fibers hardly split and fuse nor intertwine. Apparently, hydrogels formed from HG1 consist of an entangled network of very long, thin, and stiff fibers, and the network structure is stabilized by mechanical contacts between fibers rather then specific junction zones.

A very different type of network structure is formed by surfactants like cetyl$N, N, N$-trimethylammonium tosylate (CTAT, Fig. 2). In water, CTAT first forms spherical micelles just above its critical micelle concentration $(\mathrm{cmc})$ of $0.2 \mathrm{mM}$, due to hydrophobic interactions between the long alkyl chains. ${ }^{17}$ The spherical micelles quickly transform into cylindrical micelles upon increasing the surfactant concentration. These elongated structures start to entangle around $c^{*} \approx 20 \mathrm{mM}$, leading to highly viscous solutions which, however, still exhibit gravitational flow. The morphological transition from spherical to cylindrical micelles can be attributed to an increased binding of the tosylate counter-ions to the quarternary ammonium headgroups. This leads to a decrease of the headgroup repulsion and hence an increase of the packing parameter. Fig. 3d shows a cryoTEM micrograph of a $100 \mathrm{mM}$ solution of CTAT, which consists of a dense network of highly entangled, thin cylindrical micelles. Their diameter is very uniform with a mean value of 5-6 nm, which nicely agrees with the literature. ${ }^{18}$ Moreover, the persistence length is only 20-30 times the diameter of the micelles and many bifurcations can be observed..$^{19}$ These observations are in nice agreement with the general view of cylindrical micellar networks as highly dynamic equilibrium networks which are stabilized by specific interactions or junction zones between the cylindrical micelles.

Binary systems consisting of HG1 and CTAT in water could be prepared by just mixing the hydrogels and micellar solutions at room temperature, but macroscopically homogeneous samples could only be obtained by excessive vortexing. Moreover, the binary samples prepared in this way only converted slowly to a homogeneous gel state, typically within a few hours depending on the concentration. A much easier and reliable way to obtain macroscopically homogeneous aqueous samples is to exploit the thermoreversible nature of HG1 hydrogels. Thus, HG1 and CTAT could be dissolved together in water by heating at $T>$ $T_{\mathrm{GS}}$ (temperature of gel-sol transition) of HG1. After cooling to room temperature the samples were inspected for gel formation. Homogeneous, transparent gels were obtained if the HG1 concentration exceeded the minimum gelation concentration (cgc), in the presence of CTAT, at concentrations below and above the cmc and $c^{*}$. These observations suggest that at least gel formation by HG1 is compatible with the presence of spherical or cylindrical micelles. Fig. 3e and $3 \mathrm{f}$ show cryoTEM micrographs of gels of HG1 with CTAT above $c^{*}$. Elongated, almost straight fibers with a diameter of 5-25 $\mathrm{nm}$ are visible, reminiscent of the HG1 fibers morphology. In the areas in between these fibers, much thinner, curved fibers with a uniform diameter of $5 \mathrm{~nm}$ also can be distinguished clearly, similar to CTAT wormlike micelles. Moreover, both types of fibers appear to be homogeneously distributed over the samples and form a network structure of their own. Apparently, simple mixing of these two types of networks forming agents HG1 and CTAT leads to the formation of interpenetrating networks by self-assembly, a so-called SAIN. The similarity of both types of fibers to those observed in samples of the individual components strongly suggests that they originate from the self-assembly of HG1 and CTAT, respectively. At larger magnifications, however, the thicker fibers ascribed to HG1 seem to have a different fine structure then those in gels of HG1 alone. At this stage it is not clear whether this fine structure points to a different fiber structure, e.g. due to interactions with CTAT or to the overlay of the thicker fiber with much denser network of the thinner fibers.

These results clearly show that the morphological features of fibers of the separate components are largely preserved in the mixed systems, which suggests that selfassembly of HG1 and CTAT proceeds independently. However, the slight changes of fiber morphology of the thicker, presumably HG1, fibers might point to the

This journal is (c) The Royal Society of Chemistry 2009 Faraday Discuss., 2009, 143, 345-357 | 351 


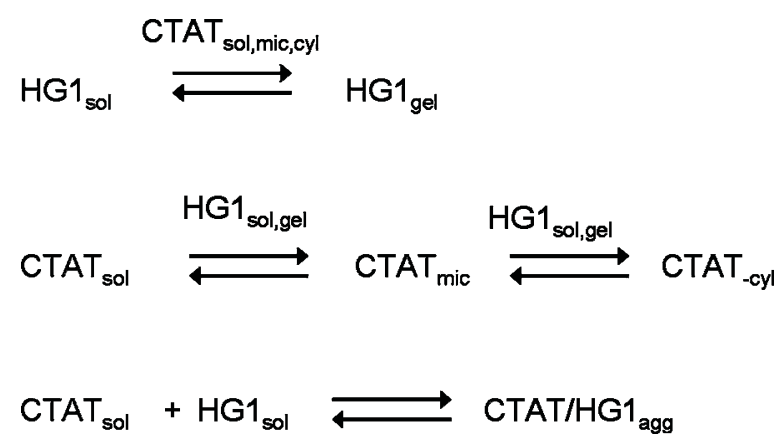

Scheme 1 Existence of different aggregation states for (1) the hydrogelators that can exist in a monomeric or gel state in presence of surfactants, and (2) the surfactants that can exist in a monomeric state or in a micellar state (spherical or cylindrical). Situations 1 and 2 represent the case of truly orthogonal processes where the different aggregation states are not affected by the other component, while the last situation depicts the possible formation of mixed aggregates.

involvement of CTAT in HG1 fiber formation. Moreover, these conclusions are based on the assumption that the thicker and thinner fibers originate from HG1 and CTAT, respectively, and furthermore, the morphological investigations do not give insights to intermolecular interactions at the molecular or micellar level. In order to address the question of if self-assembly of the hydrogelator and surfactant are true orthogonal processes, one has to investigate the self-assembly phenomena in these mixed systems in more detail. Already a first glance at mixed systems of gelators and surfactants showed that this is a challenging task because the existence of different aggregation states for each component leads to many possible interactions (Scheme 1). A similar situation will exist in any other binary self-assembling system, and will become even more complicated with increasing number of components and/or aggregation states. Fortunately, in order to address the question of whether the self-assembly processes are orthogonal or not, it is sufficient to qualitatively assess whether the equilibria are affected by the presence of another component or not, rather then to investigate each equilibrium in detail.

Because gel fibers and micelles can be considered as separate phases, the selfassembly equilibria of gelators and surfactants can, in a first approximation, be described by the pseudo-phase model. ${ }^{20}$ Hence, the free Gibbs energies for gel fiber or micelle formation from non-aggregated molecules can directly be determined from the critical aggregation concentrations (cac) or critical micelle concentrations (cmc), respectively. The cac and $\mathrm{cmc}$ of single component systems are easily measured by a wide variety of methods. For instance, a number of techniques have been implemented to obtain the cmc of surfactants, ${ }^{20}$ among which are surface tension measurement, dynamic light scattering and NMR (line broadening or changes in the chemical shift can occur upon micellization). However, these latter methods obviously can not be applied to systems containing HG1 in the gel state, and therefore methods which are specific for the surfactant or micellar state have to be used. Fluorescence probes for hydrophobic microdomains ${ }^{21}$ could be an option, but their fluorescence might still be affected by the gel fibers which could entrap some of them. In the specific case of HG1 and CTAT studied here, the non-ionic character of HG1 together with the ionic CTAT allows the application of conductimetry to measure the cmc (Fig. 4). Micellization is indeed usually accompanied by a sudden change in the slope of the specific conductivity as a function of the surfactant concentration, due to counter-ion condensation around the micelle, which decreases the free counter-ion concentration in the medium. ${ }^{22}$ Using this property, a value of $0.2 \pm 0.05 \mathrm{mM}$ was found for CTAT in water, which is in agreement

352 | Faraday Discuss., 2009, 143, 345-357 This journal is @) The Royal Society of Chemistry 2009 


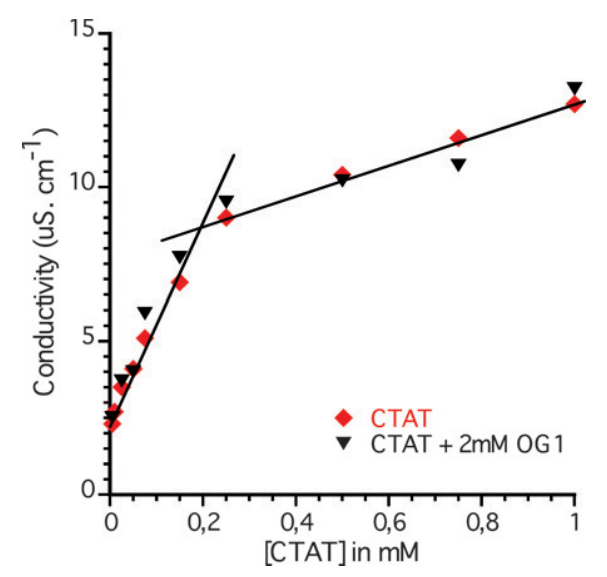

Fig. 4 Cmc determination by conductimetry of CTAT in pure water or in presence of $2 \mathrm{mM}$ HG1. Extrapolation of the two linear domains gives a cmc of about $0.2 \mathrm{mM}$ for CTAT alone, and $0.15 \mathrm{mM}$ in presence of $\mathrm{HG} 1$.

with other methods as well as the literature. Remarkably, the conductivity of CTAT solutions was hardly influenced by the presence of HG1, and a comparable cmc of CTAT in presence of gel fibers was found $(0.15 \pm 0.05 \mathrm{mM})$. It should be noted that in this experiment HG1 was present at a concentration of $2 \mathrm{mM}$, i.e. well above its cac of $0.4 \mathrm{mM}$, implying that both monomeric HG1 and HG1 fibers were present. Therefore, it can be concluded that at least micelle formation of CTAT is hardly influenced by HG1 either as non-aggregated molecules or fibrous aggregates.

In order to establish whether binary systems of HG1 and CTAT display orthogonal self-assembly one also has to assess the effect of CTAT on HG1 self-assembly, e.g. on its cac or gel-sol phase transition temperatures $\left(T_{\mathrm{GS}}\right)$. Although it is straightforward to estimate the higher limit of the cac by simple, qualitative gelation experiments like the inverted tube test, precise determinations of the cac are much more difficult and time consuming because fiber and gel formation involve a nucleation step. On the other hand, the gel-sol phase transition temperatures can easily be determined by the dropping ball method. ${ }^{23}$ This critical temperature increased steeply with the concentration of HG1 at lower concentrations, until a limiting value of approximately $130{ }^{\circ} \mathrm{C}$ was reached at concentrations of $\mathrm{HG} 1$ above $10 \mathrm{mM}$
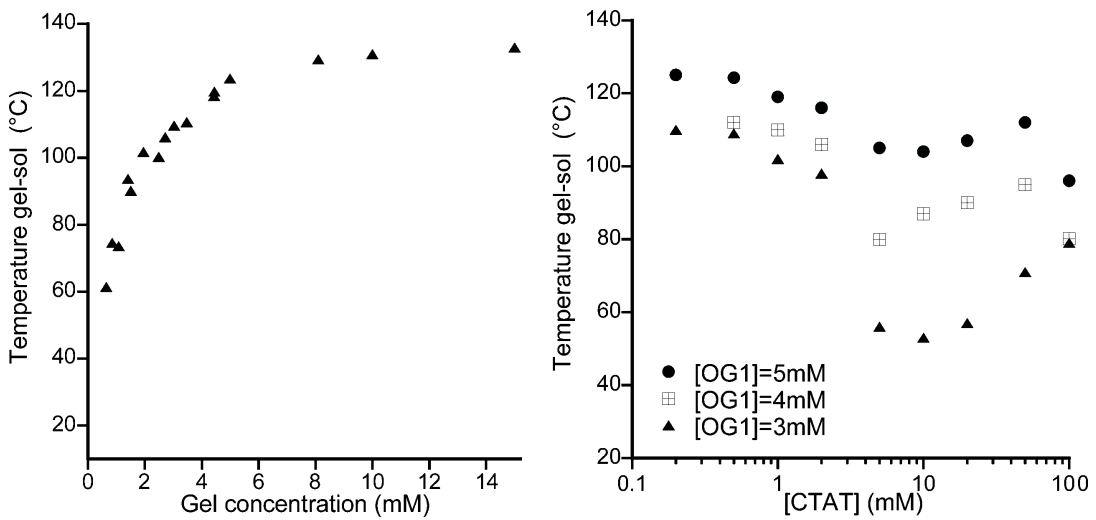

Fig. 5 (left) Temperature of gel-sol transition $\left(T_{\mathrm{GS}}\right)$ of $\mathrm{HG} 1$ as a function of its concentration. (right) Effect of CTAT concentration on the $T_{\mathrm{GS}}$ of HG1 at 3,4 or $5 \mathrm{mM}$.

This journal is ๑ The Royal Society of Chemistry 2009 Faraday Discuss., 2009, 143, 345-357 | 353 
(Fig. 5). Extrapolation of the $T_{\mathrm{GS}}$ curve to room temperature indicated a minimum gelation concentration of $0.4 \mathrm{mM}$. Such phase behaviour is typical for LMW gelators and the $T_{\mathrm{GS}}$-concentration curves have often been analyzed by the van't Hoff relationship for the melting temperature and enthalpy. ${ }^{24}$ However, it should be noted that the dropping ball method applied here monitors the gel-to-sol phase transition. This transition can have different origins: (i) dissociation of the junction zones stabilizing the network and (ii) a decrease of the fiber concentration below a critical fiber concentration necessary for maintaining a self-supporting network, e.g. due to melting of individual fibers. These processes can coincide or take place independently, but in both cases fibers are still present at the observed $T_{\mathrm{GS}}$, and therefore this temperature underestimates the actual melting temperature for a specific gelator concentration..$^{25}$

While the presence of self-assembled gel fibers did not seem to significantly alter the cmc of the CTAT surfactants, reciprocally, the presence of surfactants in the hydrogels led to a significant destabilization of the gel network, manifested by a decrease of $T_{\mathrm{GS}}$ (Fig. 5). This effect was more pronounced at low concentrations of gel ( $3 \mathrm{mM} \mathrm{HG} 1)$ than at higher concentration $(\mathrm{HG} 1=5 \mathrm{mM})$ and occurred as soon as the first micelles of CTAT were formed. However, the $T_{\mathrm{GS}}$ typically increased back to its original value in water when the CTAT concentration was further increased, i.e. when the elongated micelles were progressively growing to finally entangle. The first decrease in the $T_{\mathrm{GS}}$ might be explained by a partial dissolution of monomers in the micelles, leading to a more limited number of gelator available for gel formation. In that case, a more significant shift in the cmc of the CTAT would be expected. The other possibility is that a small fraction of surfactants favours end-capping of the fibers, making them shorter and thus resulting in more fragile gels. While the outside of the fibers is mostly covered by ethylene glycol tails, the extremity of the fibers may indeed expose their more hydrophobic core possibly interacting with the hydrophobic tails of the surfactants. Such behaviour was in fact observed with a similar self-assembled gelator in the presence of spherical micelles. The further increase in $T_{\mathrm{GS}}$ with increasing CTAT concentrations is not clear yet. This effect might be due to the presence of more and more entangled micelles that could act as a mechanical support for the stiff gel fibers and thus prevent the gel to collapse. The other possibility is that the surfactant-surfactant interactions become stronger as the surfactant packing parameter increases, limiting as a consequence the surfactant-gel interactions.

In conclusion, the change in the critical parameters for gel formation suggests that a fraction of gelators and surfactants most probably interact at a molecular or supramolecular level. The fact that these binary systems are prepared from isotropic solutions and not simply mixed corresponds, of course, to the most drastic conditions to favour interactions between monomers of each entity. Different results may have been observed by only mixing the two species directly in an aggregated state. Still, even in conditions where the two components are given high opportunities to interact first from monomeric states, it appears that these molecules have a high tendency to self-sort in two different self-assembled networks, with characteristics very close to the original networks in water.

The development of such interpenetrating networks, with distinct rheological properties may also lead to the emergence of new viscoelastic properties, unattainable using the individual components separately. The rheological responses of the two distinct networks, as well as their behaviour when mixed together, were therefore investigated using oscillatory shear experiments. For each case, Fig. 6 shows the frequency dependence of the dynamic moduli, which provides insight into the relaxation and lifetime of the connections between the units forming a network. Frequency sweeps at $25^{\circ} \mathrm{C}$ were performed within the common linear viscoelastic region of the two networks $(\gamma=0.5 \%)$. For a $100 \mathrm{mM}$ CTAT sample, in the lowfrequency region, $G^{\prime}$ and $G^{\prime \prime}$ were found to scale with frequency with exponents of 2 and 1, respectively. At higher frequencies, $G^{\prime}$ and $G^{\prime \prime}$ crossed over at a characteristic

354 | Faraday Discuss., 2009, 143, 345-357 This journal is @) The Royal Society of Chemistry 2009 

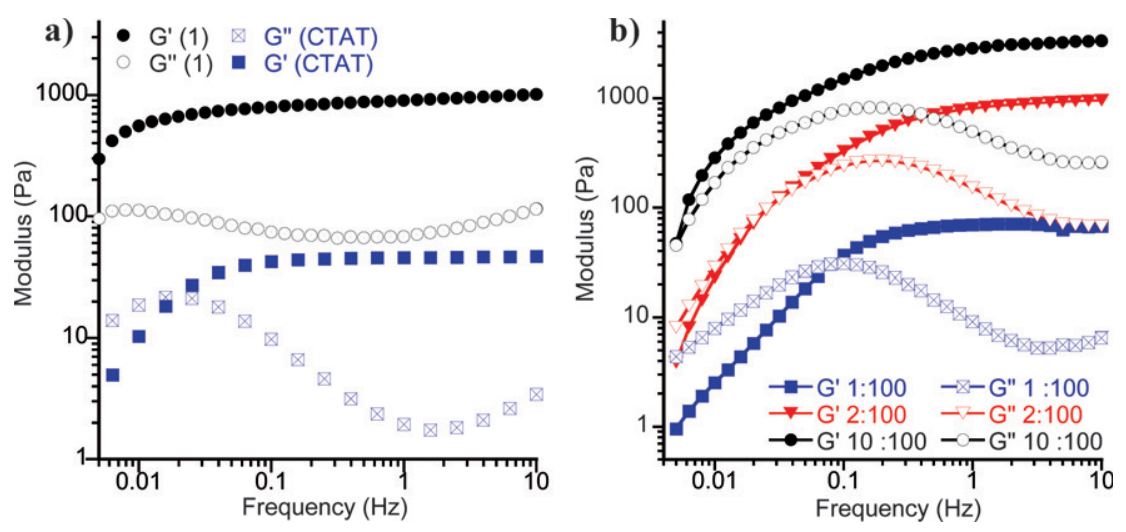

Fig. 6 (a) Moduli frequency dependence $\left(G^{\prime}\right.$ and $\left.G^{\prime \prime}\right)$ of a $100 \mathrm{mM}$ CTAT solution in water (wormlike micelles) or a hydrogel of HG1 at $2 \mathrm{mM}$ in water. (b) Dynamic moduli $G^{\prime}$ (elastic) and $G^{\prime \prime}$ (viscous) as a function of frequency of a mixed system of cylindrical micelles (CTAT, $100 \mathrm{mM}$ ) and gel fibers of HG1 at $1 \mathrm{mM}, 2 \mathrm{mM}$, and $10 \mathrm{mM}$ respectively ([gelator]/[surfactant] $=1: 100,2: 100$ and $10: 100)$.

frequency whose reciprocal corresponds to the main relaxation time (or disentanglement time) of the system, $\tau_{\mathrm{R}}$. A value of about $40 \mathrm{~s}$ was obtained at $25^{\circ} \mathrm{C}$, which is in agreement with literature. ${ }^{26}$ Such behaviour is very characteristic of viscoelastic solutions of entangled cylindrical micelles. ${ }^{27}$ By contrast, the gelators under study displayed a frequency response typical for gels with more permanent bonds, ${ }^{28}$ as indicated by a small frequency dependence and $G^{\prime}$ being about 10 times higher than $G^{\prime \prime}$. Indeed, for a gel concentration of only $2 \mathrm{mM}, G^{\prime}$ and $G^{\prime \prime}$ displayed a plateau at around $1000 \mathrm{~Pa}$ and $100 \mathrm{~Pa}$, respectively, over a wide range of frequencies $(0.05-10 \mathrm{~Hz})$, indicating a predominantly elastic response. The values of the plateaux reached by $G^{\prime}$ and $G^{\prime \prime}$ depended on the concentration of HG1: $G^{\prime}$ and $G^{\prime \prime}$ stabilized around $80 \mathrm{~Pa}$ and $10 \mathrm{~Pa}$ respectively for a $1 \mathrm{mM}$ solution of HG1, while values of $4000 \mathrm{~Pa}\left(G^{\prime}\right)$ and $500 \mathrm{~Pa}\left(G^{\prime \prime}\right)$ were measured for $10 \mathrm{mM}$ gels. Overall, the absence of a relaxation time, together with higher values of $G^{\prime}$ and $G^{\prime \prime}$ for hydrogel make this self-assembled network very different from solution of wormlike micelles.

Interestingly, when these two types of networks were combined, an intermediate and adjustable rheological response was observed depending on the concentration of HG1 in a CTAT solution (Fig. 6b). A $100 \mathrm{mM}$ CTAT solution in presence of $1 \mathrm{mM}$ of HG1 behaved mostly as a viscoelastic solution of entangled micelles. The values reached by $G^{\prime}$ and $G^{\prime \prime}$ were comparable to those observed with a pure solution of CTAT at the same concentration. The relaxation time measured was however smaller $\left(\tau_{\mathrm{r}} \sim 10 \mathrm{~s}\right)$, indicating a faster disentanglement of the micelles. When the concentration of HG1 was increased to $2 \mathrm{mM}$ in a $100 \mathrm{mM}$ CTAT solution, a viscous behaviour at low frequencies was still observed $\left(G^{\prime}<G^{\prime \prime}\right)$ followed by a more elastic response at high frequencies. Interestingly, the measured response was directly a combination of the two distinct behaviours of CTAT and HG1 alone. The response at low frequencies had a significant overlap with the typical response of CTAT micelles at the same concentration; while the response of the binary mixture at high frequencies appeared to be mostly governed by the quasi-elastic network of HG1. Increasing the concentration of HG1 further to $10 \mathrm{mM}$ in CTAT solution resulted in a predominantly elastic behaviour. The rheological properties of the binary mixtures appeared thus to be easily tuneable from viscoelastic systems to more elastic with increasing concentrations of HG1, even at very low ratios and without any macroscopic phase separation. Apparently, the presence of stiff fibers of HG1 reinforces the otherwise soft gels of CTAT, whereas, reciprocally, rigid gels of HG1 become more dynamic due to the presence of cylindrical micelles of CTAT.

This journal is ( ) The Royal Society of Chemistry 2009 Faraday Discuss., 2009, 143, 345-357 | 355 


\section{Summary}

So far, the preparation of interpenetrating network gels by self-assembly approaches was doomed to fail because the conventional polymers and surfactant building blocks either phase separate or form mixed assemblies, respectively. In this work we showed that non-covalent interpenetrating networks can be easily prepared by the orthogonal self-assembly of small molecular hydrogelators and surfactants. The first studies showed that these self-assembled IPN have a number of intriguing properties. For instance, the presence of two coexisting networks offers new possibilities for compartmentalization, and will allow one to adjust the viscoelastic properties between 'soft' and 'hard' gels. The non-covalent character of such IPN makes their formation fully reversible, which can be exploited for dual responsive systems. Most interestingly, self-assembled IPN can also act as a very primitive, yet unique, model for biological interpenetrating networks like the extracellular matrix and the cytoskeleton, and thereby contribute to our understanding of these very complex systems.

\section{Acknowledgements}

This work was supported by Nanoned/STW and the Netherlands Organization for Scientific Research (NWO).

\section{References}

1 G. M. Whitesides, J. P. Mathias and C. T. Seto, Science, 1991, 254, 1312.

2 G. M. Whitesides and B. Grybowski, Science, 2002, 295, 2418; M. Antonietti and G. A. Ozin, Chem.-Eur. J., 2004, 10, 28.

3 J. N. Israelachvilli, Intermolecular and surfaces forces, 2nd edn, Academic Press, New York, 1992.

4 D. R. Karsa, Industrial applications of surfactants IV, Woodhead Publishing Limited, 1999, Cambridge; Y. Yamamoto, T. Fukushima, Y. Suna, N. Ishii, A. Saeki, S. Seki, S. Tagawa, M. Taniguchi, T. Kawai and T. Aida, Science, 2006, 314, 1761.

5 R. G. Weiss and P. Terech, Molecular gels - Materials with self-assembled Fibrillar Networks, Springer, Dordrecht, The Netherlands, 2006; S. Jain and F. S. Bates, Science, 2003, 300, 460; D. E. Discher and A. Eisenberg, Science, 2002, 297, 967; S. Zhang, Nat. Biotechnol., 2003, 21, 1171; K. Velonia, A. E. Rowan and R. J. M. Nolte, J. Am. Chem. Soc., 2002, 124, 4224.

6 Z. Li, E. Kesselman, Y. Talmon, M. A. Hillmyer and T. P. Lodge, Science, 2004, 306, 98 101; H. Cui, Z. Chen, S. Zhong, K. L. Wooley and D. J. Pochan, Science, 2007, 317, $647-$ 650; A. L. Parry, P. H. H. Bomans, S. J. Holder, N. A. J. M. Sommerdijk and S. C. G. Biagini, Angew. Chem., Int. Ed., 2008, 47, 8859-8862.

7 A. Aggeli, I. A. Nyrkova, M. Bell, R. Harding, L. Carrick, T. C. B. McLeish, A. N. Semenov and N. Boden, Proc. Natl. Acad. Sci. U. S. A., 2001, 98, 11857; R. Oda, I. Huc, M. Schmutz, S. J. Candau and F. C. MacKintosh, Nature, 1999, 399, 566; H. Dong, S. E. Paramonov, L. Aulisa, E. L. Bakota and J. D. Hartgerink, J. Am. Chem. Soc., 2007, 129, 12468; D. Papapostolou, E. H. C. Brornley, C. Bano and D. N. Woolfson, J. Am. Chem. Soc., 2008, 130, 5124.

8 S. Bhuniya and B. H. Kim, Chem. Commun., 2006, 1842; J. D. Hartgerink, E. Beniash and S. I. Stupp, Science, 2001, 294, 1684.

9 O. Ikkala and G. ten Brinke, Science, 2002, 295, 2407.

10 M. Boltau, S. Walheim, J. Mlynek, G. Krausch and U. Steiner, Nature, 1998, 391, 877.

11 T. Kato, Science, 2002, 295, 2414.

12 A. Heeres, C. van der Pol, M. Stuart, A. Friggeri, B. L. Feringa and J. H. van Esch, J. Am. Chem. Soc., 2003, 125, 14252; A. Brizard, M. Stuart, K. van Bommel, A. Friggeri, M. de Jong and J. van Esch, Angew. Chem., Int. Ed., 2008, 47, 2063.

13 A. M. Brizard and J. H. van Esch, Soft Matter, 2009, 5, 1320.

14 L. H. Sperling, Interpenetrating polymer networks and related materials, Plenum, New York, 1981; Y. H. Lim, D. Kim and D. S. Lee, J. Appl. Polym. Sci., 1997, 64, 2647; A. Gutowska, Y. H. Bae, H. Jacobs, J. Feijen and S. W. Kim, Macromolecules, 1994, 27, 4167; Y. Liua and M. B. Chan-Par, Biomaterials, 2009, 30, 196; H. Katono, K. Sanui, N. Ogata, T. Okano and Y. Sakurai, Polym. J., 1991, 23, 1179.

356 | Faraday Discuss., 2009, 143, 345-357 This journal is @ The Royal Society of Chemistry 2009 
15 Advances in Interpenetrating Polymer Networks, ed. D. Klempner and K. C. Frisch, Technomic: Lancaster, PA, 1994; J. J. M. Halls, C. A. Walsh, N. C. Greenham, E. A. Marseglia, R. H. Friend, S. C. Moratti and A. B. Holmes, Nature, 1995, 376, 498.

16 K. J. C. van Bommel, C. van der Pol, I. Muizebelt, A. Friggeri, A. Herees, A. Meetsma, B. L. Feringa and J. H. van Esch, Angew. Chem., Int. Ed., 2004, 43, 1663-1667.

17 J. F. A. Soltero and J. E. Puig, Langmuir, 1995, 11, 3337.

18 L. M. Walker, Curr. Opin. Colloid Interface Sci., 2001, 6, 451.

19 In solution, the persistence length is probably even shorter, since the preparation of the grids for cryoTEM most likely favours alignment of the elongated micelles, due to shear forces when blotting away the excess of liquid. For a critical analysis of this method applied to wormlike micelles see: Y. I. Gonzalez and E. W. Kaler, Curr. Opin. Colloid Interface Sci., 2005, 10, 256.

20 D. F. Evans and H. Wennerström, The colloidal domain, VCH, Weinheim, 1994.

21 M. C. A. Stuart, J. C. van de Pas and J. B. F. N. Engberts, J. Phys. Org. Chem., 2005, 18, 929; J. Aguiar, P. Carpena, J. A. Molina-Bolívar and C. Carnero Ruiz, J. Colloid Interface Sci., 2003, 258, 116; C. Carnero Ruiz, Colloid Polym. Sci., 1995, 273, 1033.

22 P. Mukerjee, J. Phys. Chem., 1962, 66, 1375; A. K. Jain and R. P. B. Singh, J. Colloid Interface Sci., 1981, 81, 536.

23 Takahashi, M. Sakai and T. Kato, Polym. J., 1980, 12, 335-341.

24 P. Terech and R. G. Weiss, Chem. Rev., 1997, 97, 3133.

25 P. Terech, C. Rossat and F. Volino, J. Colloid Interface Sci., 2000, 227, 363.

26 J. F. A. Soltero and J. E. Puig, Langmuir, 1996, 12, 2654.

27 J. F. Berret, in Rheology of worm-like micelles in Molecular gels - Materials with selfassembled Fibrillar Networks, Springer, Dordrecht, The Netherlands, 2006, chapter 19.

28 P. Terech, A. Coutin and A. M. Giroud-Godquin, J. Phys. Chem. B, 1997, 101, 6810; P. Terech, D. Pasquier, V. Bordas and C. Rossat, Langmuir, 2000, 16, 4485; J. Brinksma, B. L. Feringa, R. M. Kellogg, R. Vreeker and J. H. van Esch, Langmuir, 2000, 16, 9249.

This journal is ( ) The Royal Society of Chemistry 2009 Faraday Discuss., 2009, 143, 345-357 | 357 